\title{
RECOGNITION OF TRAFFIC SIGNS APPLYING NEURAL NETWORK
}

\author{
Zsolt Barnabás NEUROHR ${ }^{1}$, Edit TÓTHNÉ LAUFER ${ }^{2}$ \\ ÓbudaUniversity. Donát Bánki Faculty of Mechanical and Safety Engineering, Budapest, Hungary \\ ${ }^{1}$ zsoltbarni@gmail.com \\ ${ }^{2}$ laufer.edit@bgk.uni-obuda.hu
}

\begin{abstract}
Artificial intelligence is one of the most dynamically developing areas of science today. Although it is not yet an integral part of our lives to use artificial intelligence solutions, it can be seen in terms of development, that it will become available to everyone in the coming decades, and not be exclusive for the richest. An important part of artificial intelligence research are the so-called soft calculation methods, the most important of which are fuzzy logic, genetic algorithms and neural networks. In this article, the authors present a method of identifying certain traffic signs with the help of the neural network.
\end{abstract}

Keywords: image processing, neural network.

\section{Introduction}

Traffic signs have come to play an important role in everyday traffic control, in order to avoid traffic accidents. Therefore, the recognition and interpretation of traffic signs is essential.

There are many applications which can display different objects. These include segmentation, the Kalman-filter, or the Moravec corner point detector.

Most of the image recognition methods use soft computing methods, including the neural network, based on their ability to teach.

\section{Operation of the neural network}

\subsection{Structure of the Neural Network}

The structure of the artificial neural network follows the organization of the human brain. Different parts of the human brain are responsible for vision, hearing, sense of smell, and these various areas of activity cannot replace each other. It follows that an artificial neural network is created to solve a particular problem.

Thanks to the parallel design of the network and the large number of small counter units which connected to each other, a high operating speed can be achieved. The neural network contains hidden layers between an input and an output layer, as shown in Figure 1. The layers are connected to each other via neurons. Each connection is provided with a weight factor, which is assigned a value based on efficiency [1].

The network is not programmed, but is taught by large a numbers of patterns. These stored data are divided in the network, so the network is error tolerant, if you remove or modify an item, it will still work excellently.

There are three conditions for creating a network. The first includes computing units, the socalled neurons, the second is the topology of network interconnection, and finally, the algorithms that applies the teaching rules, which are responsible for setting the weight factors.

\subsection{The structure and the operation of the base neuron}

The neuron calculates inputs from other neurons as a weighted sum. The result is interpreted in the next step by using the transfer functions as illustrated in Figure 2., where $x_{i}$ is the input $i, w_{i}$ is the weight factor, $f(x)$ is the transfer function.

Such a function may be, for example, the unit function. If the value is less than or equal to zero, 
then the output will be zero, so no reaction will occur. However, if it is higher than zero, the output will take the value of one.

Other typical transfer functions may be linear and logistic [1].

\subsection{The process of teaching the network}

The network can be considered as a black box, the input has a specific output, a target value. Each layer in the network takes and transforms the data from the previous layer and then transmits to the next layer.

While learning from layer to layer, the network increases the complexity and detail of the data. The net learns directly from the data, the authors have no influence on what details of the data will be learned.

Accordingly, a learning pattern is provided for the input of the network and its output calculated, finally it is compared with the target value. If inadequate results have been obtained, the weight factors should be tuned until the appropriate efficiency is achieved. This is called controlled or supervised teaching, when the net learns the inputs and outputs which are generated by the pairs of samples.

The network can be considered functional if we obtain the right target values for test samples that do not contain the teaching patterns [1].

\subsubsection{Teaching rules}

Two teaching rules are known, one of which is the Hebb rule, stating that the weight factor between two connected neurons is increased if they are active or inactive at the same time. In such cases the connection will be strengthened, while in the other cases it will be downgraded.

The other rule referred to is the delta rule, when the weight value is modified to the optimal val-

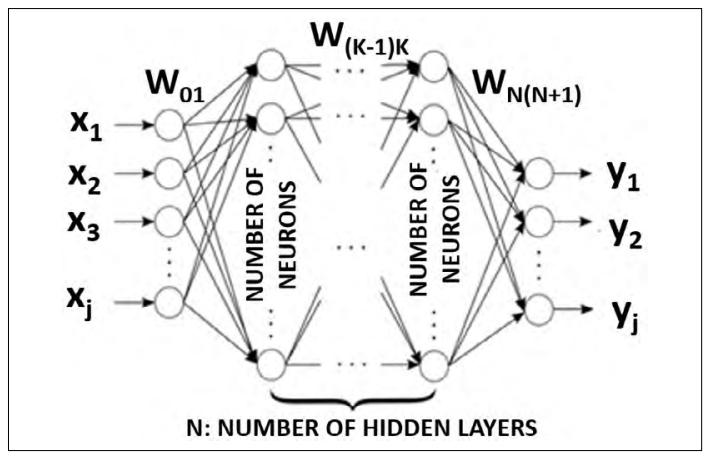

Figure 1. The topology of the neural network ue by algorithm, while minimizing the difference between the output and the desired target value.

\section{Case study}

\subsection{Test environment}

The purpose of the study is to identify four different traffic signs (stop, crosswalk, give way, no entry).

The toolbars provided by the MATLAB program are used to solve the task. The Deep Learning Toolbox is used to create and teach the neural network. The teaching of the network is realized by the method of supervised teaching.

\subsection{The creation of the database}

As a first step, a database is created with images arranged in subfolders by sign type. Each image has its own folder name. Then, the images are randomly divided into two databases. One database was used as a teaching sample, while the other for testing the image recognition efficiency of the network.

\subsection{The creation of the network}

The created network consists of 14 hidden layers between the input and output layers which recognize the features. The structure of the network is shown in Figure 3.

The input layer of the network consists 3600 neurons, each of which corresponds to one pixel of a $60 \times 60$ upstream input image.

During the study, it was confirmed that using a higher resolution image size did not contribute to more accurate image recognition, but slowed down the operation of the network. The first hidden layer is the so-called convolutional layer, where the neurons process only a small part of the input image. The operation performed by the neurons is very similar to the filters known from

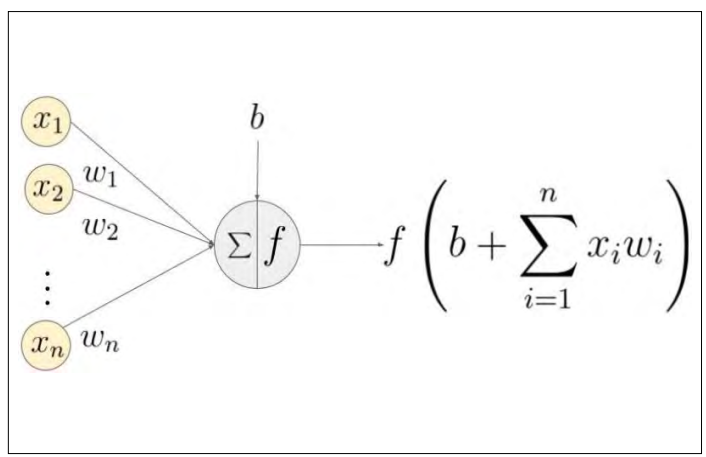

Figure 2. The structure of the base neuron 
image processing. We expect onvolutional neurons to be able to learn some more or less abstract concepts (e.g. detection of an edge). Generally, a single neuron is not enough for this, so more neurons are taught in the same input position in parallel. Generally, a single neuron is not enough for this, therefore more neurons are taught in the same input position parallel. In this case 40 neurons are used, which are to provide 40 output matrixes [2]. The so-called ReLu activation function is used at the output. (Figure 4.) . Its function is partly biology-based and analogous to how neurons are activated or not. If the output value exceeds the threshold value, the function returns itself, whereas, if the threshold value is not exceeded, then the output will clearly be zero [3].

The further and deeper the process proceeds, the less it becomes necessary to preserve the smaller, finer details. For example, if the "edge detector" indicates that an edge is found, then its exact position will no longer be needed in the deeper layers. Therefore, in the next layer, the activated outputs is made simpler. By building the hidden layers mentioned above, increasingly abstract concepts can be gained. In the present case, it turned out to be enough to repeat the above operation three

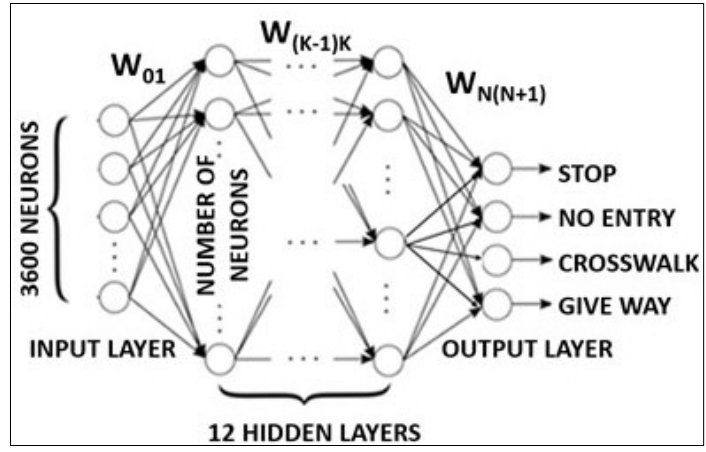

Figure 3. The topology of the created neural network

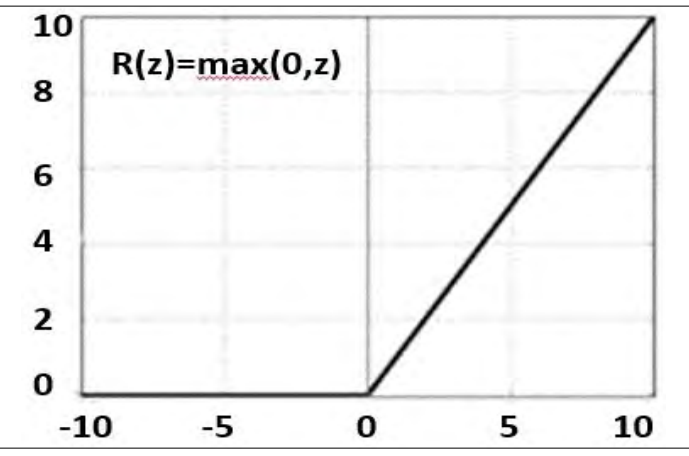

Figure 4. Image and formula of the ReLu function times to obtain the appropriate efficacy. The network contains 14 hidden layers.

Finally, the output layer is assigned, which conducts the classification and consists of four neurons corresponding to the four classes.

The teaching of the network is accomplished with a gradient based extreme value search method and supervised teaching.

Using the present methods, the created network has been able to recognize the signs with an 85-90 percent efficiency following a learning period of one and a half minutes.

\subsection{Alexnet}

For comparison, the teaching process is also conducted on the Alexnet network, developed by Alex Krizhevsky, which has already been able to recognize the signs with a 99 percent efficiency by learning for seven minutes.

The difference may be that while the created net is developed by less than three hundred images, Alexnet is trained by millions of images, and Alexnet's structure consists of 25 layers in contrast to the created net, which consists of 14 layers [4].

The following matrixes can be used to illustrate the effectiveness of networks, in which blue indicates diagonals, being the number of correct solutions, the number of faults is represented by pale red. From the left-hand matrix, it is clear that Alexnet has been able to recognize almost all classes of test images, while the created network failed to detect the class of images in several cases.

Based on the comparison (Figure 5.), it is stated that the network can become more efficient if it is built up of several hidden layers and taught with more data.

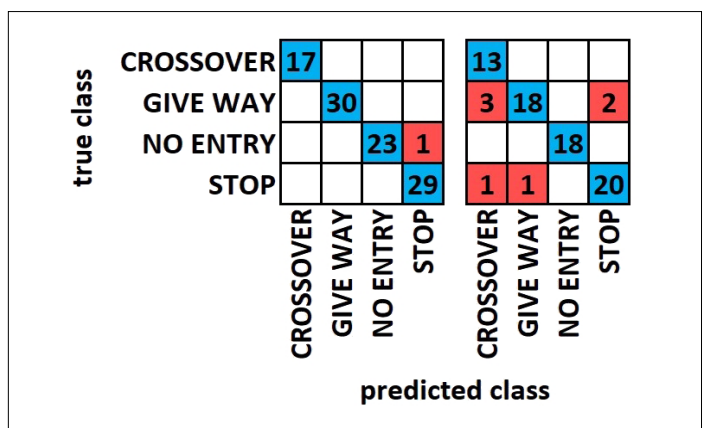

Figure 5. Comparison of Alexnet and the created network 


\section{Conclusion}

The aim of this article was to build a neural network for recognizing traffic signs. The network was created in the Matlab environment with the help of the Deep Learning Toolbox. Based on a practice test, the network was able to classify the traffic signs with 85-90 percent efficiency by one and a half minutes learning. For safety reasons, it is necessary to improve the efficiency, which can be achieved by incorporating additional layers or by using a larger set of teaching.

\section{Acknowledgment}

Supported by the Donát Bánki Special Collage, and ÚNKP-18-1 New National Excellence Program of the Ministry of Human Capacities.

\section{References}

[1] Altrichter M., Horváth G. et. al.: Neurális hálózatok. Hungarian Edition Panem Könyvkiadó Kft., Budapest, 2006.

[2] Krizhevsky A., Hinton G.: Learning multiple layers of features from tiny images." Master's Thesis. University of Toronto, Toronto, Canada, 2009.

[3] Nair V., Hinton G. E.: Rectified linear units improve restricted boltzmann machines. In Proc. 27th International Conference on Machine Learning, 2010.

[4] Krizhevsky, A. I. Sutskever, et. al.: ImageNet Classification with Deep Convolutional Neural Networks. Advances in Neural Information Processing Systems. Vol 25, 2012. 